\title{
Caracterização da Gravidez com Insuficiência Istmocervical
}

\author{
Characterization of Pregnancy with Cervical Incompetence
}

Liliane Costa Rodrigues, Rosiane Mattar, Luiz Camano

\begin{abstract}
RESUM0
Objetivo: identificar características comuns às portadoras de insuficiência istmocervical (IIC) que serviriam para reconhecê-las precocemente e auxiliariam no seguimento do ciclo gravídicopuerperal.

Pacientes e Métodos: cinqüenta gestantes portadoras de IIC submetidas à circlagem uterina segundo a técnica de McDonald modificada foram analisadas em estudo descritivo observacional. Por meio dos dados dos prontuários, foram estudadas as seguintes variáveis: idade, antecedentes obstétricos, momento do diagnóstico de IIC, aspectos relativos à circlagem $e$ incidência de prematuridade.

Resultados: em média, a idade materna foi de 29,2 anos e o número de gestações anteriores foi de 3,7. Quanto aos antecedentes obstétricos, tivemos 189 gestações anteriores; em 18 delas já se tinha o diagnóstico de IIC e a circlagem havia sido realizada, tendo resultado em 18 crianças vivas (8 nasceram prematuramente e 10 no termo). Das 171 prenhezes prévias em que o diagnóstico de IIC não estava firmado, 90 evoluíram para abortamentos, 68 foram partos pré-termos e 13 de termo. Em relação ao momento do diagnóstico de IIC, 30 (60\%) pacientes tiveram-no firmado no intervalo entre os partos e 20 (40\%) durante a gestação. A idade gestacional média em que as pacientes foram submetidas à circlagem foi de 18,2 semanas; $40 \%$ das circlagens foram de urgência e $60 \%$ eletivas. A incidência de prematuridade foi de 30\% (15/50).

Conclusão: a avaliação obstétrica cuidadosa de multigestas com perdas gestacionais recorrentes, no intervalo entre os partos e/ou no primeiro trimestre da gravidez, pode permitir o diagnóstico precoce e tratamento adequado da IIC, evitando a prematuridade.
\end{abstract}

PALAVRAS-CHAVE: Insuficiência istmocervical. Prematuridade. Circlagem. Abortamento tardio.

\section{Introdução}

A prematuridade representa ainda um dos grandes temores do obstetra. Apesar dos inúmeros avanços conseguidos pela Perinatologia, com as modernas técnicas de cuidados intensivos e o melhor conhecimento da fisiopatologia do recémnascido, os índices de morbimortalidade neonatal ainda se mantêm elevados ${ }^{1}$.

Diante desse fato, a identificação precoce e segura do trabalho de parto prematuro é importante, pois pode impedir o nascimento pré-termo

Departamento de Obstetrícia, Universidade Federal de São Paulo - Escola Paulista de Medicina

Correspondência:

Liliane Costa Rodrigues

Rua Argonautas, 294 - Vila Formosa

03360-060 - São Paulo - SP

Fone/ Fax: (11) 6211-8630

e-mail: rosiane.toco.@epm.br ou, ao menos, permitir que ele ocorra em melhores condições. A história de prematuridade anterior é importante fator de risco para recorrência na gestação atual e seu conhecimento permite diminuir os agravos decorrentes da prematuridade, por meio de medidas preventivas durante o prénatal, como medidas higienodietéticas, uso de tocolíticos e corticoterapia ${ }^{2-6}$.

Entre os principais fatores envolvidos na etiologia da prematuridade está a insuficiência istmocervical (IIC), responsável por 16 a $20 \%$ das perdas gestacionais ocorridas no segundo trimestre de gestação ${ }^{7}$. A IIC é a entidade clínica que determina a falência do sistema oclusivo da matriz, impossibilitando-a de manter-se convenientemente fechada para reter o produto da concepção até o final da gestação $0^{8,9}$.

Em vista de sua etiopatogenia e das conseqüências que acarreta, pode-se pressupor que a IIC acometeria mulheres com algumas caracte- 
rísticas em comum, o que poderia nortear a identificação precoce das portadoras desta doença. A literatura mostra que a doença é mais comum em mulheres com idade mais avançada ${ }^{10-12}$. Entretanto, Lidegaard ${ }^{11}$ chama atenção para o fato que a IIC pode ser diagnosticada em mulheres jovens, entre 15 e 19 anos.

Em relação à paridade e aos antecedentes obstétricos existe concordância entre os trabalhos realizados, em que a portadora de IIC, apresenta várias prenhezes prévias, com abortamentos, prematuridade e neomortalidade, e os melhores resultados obtidos com a realização da circlagem têm sido em gestantes com pouca paridade (menos de dois partos anteriores) ${ }^{13}$.

Quanto ao momento do diagnóstico de IIC, diversos autores são unânimes em afirmar que a melhor maneira de estabelecê-lo seria fora da gestação, baseado nos antecedentes obstétricos e exames complementares ${ }^{14}$. Alguns estudiosos referem que a época ideal para a realização da circlagem seria um momento previamente definido, como afirmam Kurup e Goldkrand ${ }^{15}$. Para outros, esta cirurgia só deveria ser indicada durante a gravidez, perante a dilatação cervical $^{16}$. Encontramos que o limite mínimo de idade gestacional apurado na literatura para indicação da circlagem foi de 10 semanas. Entretanto, Kurup e Goldkrand ${ }^{15}$ observaram que, nas cirurgias realizadas tardiamente, existe freqüentemente certo grau de dilatação cervical e exposição das membranas, que provocam maior número de complicações maternas e menor índice de sucesso.

Em relação às condições do colo uterino no momento da circlagem, os autores ${ }^{15}$ são unânimes em afirmar que a indicação pode ser eletiva ou urgente, e que o maior índice de sucesso é obtido quando o procedimento é realizado eletivamente, sem que haja alterações cervicais.

O objetivo deste trabalho é identificar características comuns às portadoras de IIC que serviriam para reconhecê-las precocemente e que auxiliariam no seguimento do ciclo gravídicopuerperal.

\section{Pacientes e Método}

Este estudo é retrospectivo, tendo sido incluídas 50 pacientes que receberam acompanhamento pré-natal no ambulatório específico para abortamento habitual do Departamento de Obstetrícia da Universidade Federal de São Paulo - Escola Paulista de Medicina (UNIFESP - EPM) no período de novembro de 1996 a dezembro de 1999. Todas receberam diagnóstico de IIC e foram submetidas a circlagem uterina à McDonald modificada.

O protocolo do serviço previa condutas diferentes se a paciente chegava grávida ao ambulatório, com o passado obstétrico fortemente sugestivo de IIC, ou quando já apresentava dilatação e esvaecimento precoces do colo uterino, sem trabalho de parto. Nesta última situação indicávamos a circlagem. Nos casos em que a história não era típica de IIC, empregávamos a ultra-sonografia seriada de colo, entre a $14^{\mathrm{a}}$ e a $28^{\mathrm{a}}$ semana. $\mathrm{O}$ intuito era observar se havia encurtamento de seu comprimento e principalmente, sob pressão praticada no fundo uterino, eventual abertura do orifício interno, o que caracterizava o diagnóstico intragestacional de IIC.

A circlagem era realizada eletivamente, entre a $12^{\mathrm{a}}$ e a $16^{\mathrm{a}}$ semana de gestação, preferencialmente ao redor da $14^{\mathrm{a}}$ semana. Porém, essa técnica podia ser efetuada mais tardiamente, até 28 semanas, em casos em que a paciente chegasse a nosso serviço numa idade gestacional mais avançada, ou quando as alterações cervicais estivessem presentes.

Considerávamos a circlagem eletiva quando o canal cervical estivesse impérvio e a urgência, quando houvesse dilatação do colo uterino ao exame ginecológico e/ou protrusão das membranas pela vagina.

Antes do procedimento, era realizado exame ultra-sonográfico para confirmar a idade gestacional, a normalidade da gravidez e avaliar o colo. Eram tratadas as vulvovaginites que podiam por acaso existir.

Não se utilizavam durante e após o ato cirúrgico agentes tocolíticos e antibióticos profiláticos. A paciente recebia alta após 6 a 12 horas do procedimento, com a orientação de retornar ao ambulatório de pré-natal quinzenalmente, ou a qualquer momento se ocorresse alguma intercorrência.

O presente estudo é descritivo e foi aprovado pelo Comitê de Ética em Pesquisa da Universidade Federal de São Paulo/Hospital São Paulo.

Tendo como base os prontuários, foram estudadas as seguintes variáveis: idade: entre 15 e 40 anos, analisadas em períodos, a cada 5 anos (15 a 20; 21 a 25; 26 a 30; 31 a 35; 36 a 40); antecedentes obstétricos: número de gestações anteriores/prematuros anteriores/abortamentos (precoces e tardios)/gestações de termo; momento do diagnóstico de IIC: fora ou durante a gravidez; aspectos relativos à circlagem: analisamos o momento da intervenção cirúrgica (oportuna/tardia) e as condições do colo uterino no momento da intervenção cirúrgica (eletiva/urgência); verificamos também a incidência de prematuridade: termo: > 37 semanas; pré-termo: < 34 semanas ou > 34 semanas a $<37$ semanas. 


\section{Resultados}

A análise das 50 portadoras de IIC submetidas à circlagem uterina à McDonald modificada nos mostrou que, em relação à idade materna, cerca de $16 \%$ das pacientes apresentavam idades mais avançadas, ao redor dos 40 anos, sendo menos freqüente o encontro de mulheres jovens (Figura 1). A média de idade das pacientes foi de 29,2 anos.
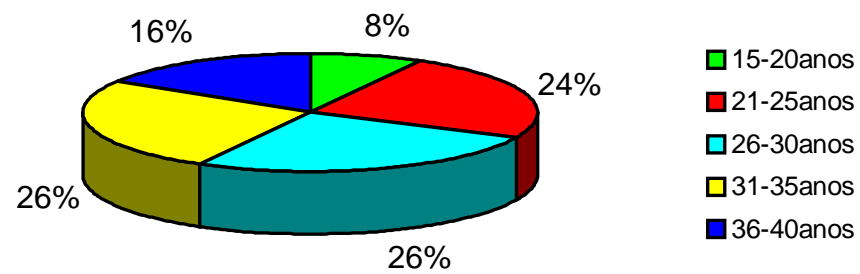

Figura 1 - Número de pacientes (\%) entre as 50 gestantes com IIC incluídas no estudo de acordo com a idade.

Em relação aos antecedentes obstétricos, as pacientes apresentaram em média 3,76 gestações anteriores (Figura 2), sendo que uma mesma paciente referia nove perdas anteriores à gravidez atual.

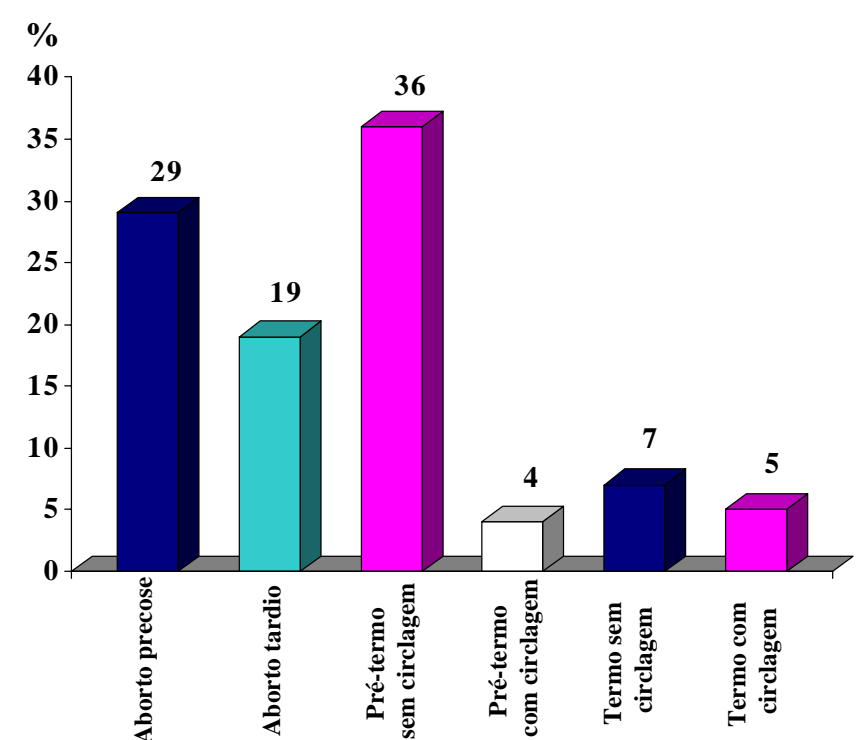

Figura 2 - Porcentagem do número de gestações anteriores e respectivos resultados nas 50 gestantes estudadas.

Observamos que as 50 gestantes analisadas referiam 189 gestações anteriores. Em 18 delas, o diagnóstico de IIC já havia sido estabelecido em gestações anteriores, e a circlagem havia sido realizada, tendo resultado em 18 crianças vivas, oito que nasceram prematuramente e dez no período de termo. Das 171 prenhezes prévias em que o diagnóstico de IIC não estava firmado, 90 evoluíram para abortamentos, 68 foram partos pré-termos e 13 foram de termo (Figura 2).

Quanto ao momento do diagnóstico de IIC, apuramos em nossa amostra que 30 pacientes (60\%) tiveram-no firmado em avaliação entre os partos. Esta forma de diagnóstico só é possivel quando a paciente é encaminhada para a realização de propedêutica por histerossalpingografia (em que se pode diagnosticar o alargamento do canal cervical com diâmetro maior ou igual a 1,0 $\mathrm{cm}$ ) e pela prova da vela 8 (observando sua progressão pelo canal cervical), após ter apresentado algum antecedente suspeito, como por exemplo perda gestacional tardia ou malformação uterina.

Estudamos alguns aspectos relacionados à circlagem uterina, como o período em que esta foi realizada, considerando oportuna, a circlagem realizada entre 12 e 16 semanas, que representou $44 \%$ da nossa população. Em 56\% dos casos, nossas pacientes chegaram em períodos considerados tardios, mesmo algumas delas já tendo o diagnóstico de IIC.

A menor idade gestacional em que se instalou a circlagem foi de 13 e a maior de 28 semanas e 3 dias. A média em que nossas pacientes foram submetidas ao tratamento foi 18,2 semanas.

As condições do colo uterino no momento da circlagem representam aspecto importante no resultado do tratamento da IIC. Em nossa casuística, 20 (40\%) foram submetidas à cirurgia com o colo uterino apresentando algum grau de dilatação, caracterizando a "circlagem de urgência”, assim denominada pelo maior risco de fracasso gestacional e prematuridade.

Em relação à idade gestacional em que ocorreram os nascimentos nas 50 gestantes com a circlagem à McDonald modificada, tivemos 35 partos a termo e 15 pré-termos (30\%), entretanto estes prematuros nasceram em idades gestacionais que possibilitaram que houvesse sobrevida do concepto (Figura 3).

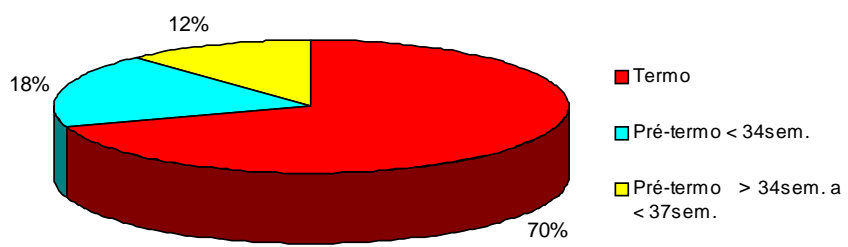

Figura 3 - Número dos nascimentos (\%) em 50 casos incluídos de acordo com a idade gestacional. 


\section{Discussão}

A IIC é doença que causa perdas gestacionais recorrentes e as portadoras desta entidade passam anos de suas vidas em gestações sucessivas que, após alguns meses, resultam em fracasso, determinando o reinício do processo, e assim, chegam a serviços especializados, mais idosas. Das 50 pacientes analisadas, $10(20 \%)$ tinham mais que 35 anos de idade, evidenciando que o retardamento do diagnóstico desta enfermidade determina que elas fiquem grávidas fora do período considerado mais oportuno para a concepção ${ }^{17}$, agravando o prognóstico materno-fetal. A média de idade de nossas pacientes foi de 29,2 anos, e é semelhante à relatada na literatura ${ }^{10,12}$.

Em relação à paridade, observamos que as mulheres portadoras de IIC passam por gestações sucessivas com vários abortos tardios e partos prematuros, sem filhos vivos, sendo em geral multigestas. Mattar et al. ${ }^{10}$ já enfatizavam que as portadoras de IIC perdiam 4,7 gestações antes do diagnóstico da IIC ser definido. Este fato é lamentável, por refletir má assistência obstétrica, retardando a resolução do problema e aumentando os riscos das gestações, que vão acontecer em pacientes mais idosas e com maior número de gravidezes anteriores.

Ressalte-se que nesse grupo de 50 gestantes com 189 gestações anteriores, observamos que de 171 gestações apenas 29 (16,9\%) resultaram em recém-nascidos vivos, significando elevado índice de perda gestacional, o que denota o drama vivido pelas portadoras de IIC. A perda reprodutiva foi de grande magnitude e 21 mulheres (42\%) de nossa casuística não tinham nenhum filho vivo. Este achado é compativel com o relatado pela literatura, que também mostra altos índices de perda gestacional e prematuridade associados ao diagnóstico de IIC ${ }^{7}$.

O diagnóstico da IIC ainda gera certa polêmica, pois não existem critérios uniformes para a sua caracterização. Vários são os autores que se baseiam na importância dos antecedentes obstétricos para firmá-1o ${ }^{15,18}$. Tendo em vista que o diagnóstico da IIC pode ser realizado durante a gravidez, e fora do período gestacional e que os melhores resultados relatados são os obtidos pelo tratamento precoce, torna-se importante estabelecer o diagnóstico desta doença no período intergestacional. Alguns autores ${ }^{19,20}$ insistem na importância do diagnóstico anterior à nova gravidez pela possibilidade de instituir tratamento cirúrgico eletivo nestes casos. Todos são unânimes em julgar fundamental o atendimento pre- coce na gravidez, para a realização da circlagem sem que tenha havido grandes alterações cervicais.

$\mathrm{Na}$ literatura tem sido considerado como período ideal para a realização da circlagem o que vai de 12 a 16 semanas $^{10,21}$. Não se indica o procedimento antes da $12^{\mathrm{a}}$, em vista da maior possibilidade de abortamento por outras causas. Após a $16^{\mathrm{a}}$ semana, por outro lado, a expansão uterina promovida pelo evoluir da gravidez determinaria a dilatação cervical com maior freqüência. Consideramos oportuna, portanto, a circlagem realizada entre a $12^{\mathrm{a}}$ e a $16^{\mathrm{a}}$ semanas, que representou $44 \%$ da nossa população. Em nossa casuística, 56\% de nossas pacientes chegaram em períodos considerados tardios. Isto acontece pelas dificuldades socioeconômicas das pacientes, que peregrinam em busca da assistência médica adequada.

A idade gestacional média em que nossas pacientes foram submetidas ao tratamento foi 18,2 semanas, considerado momento tardio pelos nossos parâmetros e os de alguns estudiosos ${ }^{22}$. Embora exista alguma polêmica sobre a época da gravidez em que deve ser feita a circlagem, os cirurgiões concordam na importância da realização da operação antes que haja dilatação do canal cervical, o que pioraria o prognóstico da gravidez. Diversos autores ${ }^{15,18,23}$ ressaltam a importância das condições do colo uterino no momento da cirurgia, considerando-o como o parâmetro mais importante para estabelecer o prognóstico.

Apesar de todas as dúvidas apontadas por alguns estudiosos sobre a validade da circlagem por via vaginal durante a gravidez, como tratamento para evitar perdas gestacionais por prematuridade, alegando que o repouso no leito poderia obter resultados semelhantes ${ }^{24,25}$, esta é a conduta mais aceita na literatura, principalmente com base nos índices de sobrevivência fetal de gestações de portadoras de IIC antes e depois de serem tratadas por este procedimento cirúrgico.

$\mathrm{O}$ índice de partos prematuros em nosso estudo foi de $30 \%$, comparando com a prevalência de prematuridade na população geral, referida como $10 \%{ }^{1}$. Os valores observados nas pacientes com IIC, mesmo tratadas com a circlagem, mostram-se muito altos, justificando a procura de métodos de identificação precoce dos casos que vão evoluir com prematuridade e assim tentar diminuir seus agravos. A avaliação obstétrica cuidadosa de multigestas com perdas gestacionais recorrentes, no intervalo entre os partos e/ou no primeiro trimestre da gravidez, pode permitir o diagnóstico precoce e tratamento adequado da IIC, evitando a prematuridade. 


\section{ABSTRACT}

Purpose: to identify characteristics common to women who suffer from cervical incompetence (CI) allowing their early identification and helping in the follow-up of the gestationalpostdelivery cycle.

Patients and Methods: fifty pregnant women suffering from $C I$ and submitted to uterine cerclage according to modified McDonald's technique were analyzed in an observational descriptive study. The following variables of the medical records were evaluated: age, preceding obstetrical aspects, time of CI diagnosis, cerclage-related aspects and preterm delivery incidence.

Results: the average age of the mothers was 29.28 years and mean number of previous gestations was 3.76. As to the preceding obstetrical aspects, 189 gestations with a CI diagnosis were reported and cerclage had been applied to 18 of them, resulting in 18 babies who were born alive ( 8 preterm deliveries and 10 term deliveries). Of the 171 previous pregnancies in which CI diagnosis was not reported, 90 progressed to abortion, 68 were preterm and 13 were term deliveries. In 30 (60\%) patients, CI was diagnosed during the intergestational interval, and 20 (40\%) during gestation. The average gestational period in which patients were submitted to cerclage was 18.29 weeks; $40 \%$ of the cerclages were emergency procedures and $60 \%$ were elective. The incidence of prematurity was $30 \%$ (15/50).

Conclusion: careful obstetrical evaluation of multiparous women, presenting recurrent miscarriages, during the intergestational interval and/or during the first trimester of pregnancy, may allow the early diagnosis and adequate treatment of $C I$, avoiding prematurity.

KEY WORDS: Cervical incompetence. Prematurity. Cerclage. Late abortion.

\section{Referências}

1. Souza E, Santos JFK, Bancher MDM, Bertini AM, Camano L. Considerações sobre a prematuridade eletiva na Universidade Federal de São Paulo Escola Paulista de Medicina. Rev Bras Ginecol Obstet 1995; 17:583-9.

2. Saling E, Schreiber M, al-Taie T. A simple, efficient and inexpensive program for preventing prematurity. J Perinat Med 2001; 29:199-211.

3. Novak-Antolic $Z$. Predicting preterm delivery and lowering very preterm delivery rate. J Perinat Med 2001; 29:469-75.

4. Sprague A, Stewart P, Niday P, Nimrod C, Walker R. Community education on preterm birth. Does it change practice? Can Fam Physician 2002; 48:72734.
5. Murphy K, Aghajafari F, Hannah M. Antenatal corticosteroids for preterm birth. Semin Perinatol 2001; 25:341-7.

6. Rosen LJ, Zucker D, Oppenheimer-Gazit V, Yagel S. The great tocolytic debate: some pitfalls in the study of safety. Am J Obstet Gynecol 2001; 184:1-7.

7. Weissman A, Jakobi P, Zahi S, Zimmer EZ. The effect of cervical cerclage on the course of labor. Obstet Gynecol 1990; 76:168-71.

8. Amaral LBD. A cirurgia da insuficiência istmocervical [tese]. Rio de Janeiro: Federação das Escolas Federais Isoladas do Estado do Rio de Janeiro; 1976.

9. Rezende J, Montenegro CAB, Barcellos JM. Abortamento. In: Rezende J, editor. Obstetrícia. $4^{\mathrm{a}}$ ed. Rio de Janeiro: Guanabara Koogan; 1982. p.563-84.

10. Mattar R, Amed AM, Camano L. O recém-nascido de mãe com insuficiência istmocervical. Rev Paul Med 1986; 104:280-2.

11.Lidegaard O. Cervical insufficiency and cerclage in Denmark 1980-1990. A registry-based epidemiological study. Ugeskr Laeger 1994; 156:7200-2.

12.Semczuk A, Kraczkowski JJ, Maleska J, Putek W, Wojcik D. Cerclage of the cervix in pregnant women with cervix incompetence in the Department of Obstetrics and Pathology of Pregnancy, School of Medicine in Lublin during the years 1982-1988. Ginekol Pol 1996; 67:325-9.

13. Waloch M. Cervical cerclage in the treatment of cervical incompetence in Zambian women. Clin Exp Obstet Gynecol 1996; 23:255-62.

14.Kassanos D, Salamalekis E, Vitoratos N, Panayotopoulos N, Loghis C, Creatsas C. The value of transvaginal ultrasonography in diagnosis and management of cervical incompetence. Clin Exp Obstet Gynecol 2001; 28:266-8.

15.Kurup M, Goldkrand JW. Cervical incompetence: elective, emergent, or urgent cerclage. Am J Obstet Gynecol 1999; 181:240-6.

16.To MS, Palaniappan V, Skentou C, Gibb D, Nicolaides KH. Elective cerclage vs. ultrasoundindicated cerclage in high-risk pregnancies. Ultrasound Obstet Gynecol 2002; 19:475-7.

17. Cecatti JG, Faúndes A, Surita FGC, Aquino MMA. O impacto da idade materna avançada sobre os resultados da gravidez. Rev Bras Ginecol Obstet 1998; 20:389-94.

18. Chanrachakul B, Herabutya Y. The epidemiology of cervical incompetence in Ramathibodi Hospital between 1982-1997. J Med Assoc Thai 1999; 82:749-53.

19.Aarnoudse JG, Huisjes HJ. Complications of cerclage. Acta Obstet Gynecol Scand 1979; 58:255-7. 
20.Mattar R. Importância do setor de assistência à insuficiência istmocervical e da ocasião do atendimento médico [tese]. São Paulo: Escola Paulista de Medicina; 1992.

21.Kokia E, Dor J, Blankenstein J, et al. A simple scoring system for the treatment of cervical incompetence diagnosed during the second trimester. Gynecol Obstet Invest 1991; 31:12-6.

22.Pritchard JA, MacDonald PC, Gant NF. Abortion. In: Pritchard JA, MacDonald PC, Gant NF, editors. Williams Obstetrics. 20 ${ }^{\text {th }}$ ed. Norwalk: Appleton \& Lange; 1997 p.579-605.
23.Matijevic R, Olujic B, Tumbri J, Kurjak A. Cervical incompetence: the use of selective and emergency cerclage. J Perinat Med 2001; 29:31-5.

24.Olatunbosun OA, al-Nuaim L, Turnell RW. Emergency cerclage compared with bed rest for advanced cervical dilatation in pregnancy. Int Surg 1995; 80:170-4.

25.Novy M J, Gupta A, Wothe DD, Gupta S, Kennedy KA, Gravett MG. Cervical cerclage in the second trimester of pregnancy: a historical cohort study. Am J Obstet Gynecol 2001; 184:1447-54.

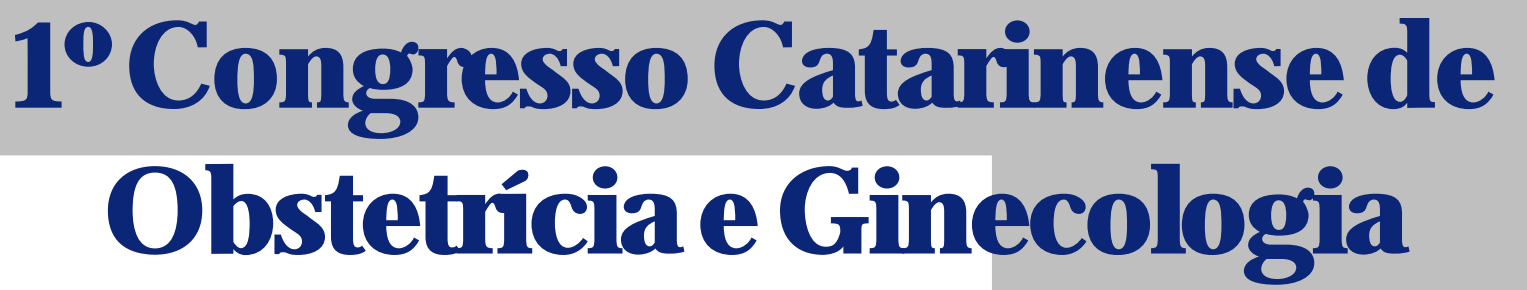

29 a 31 de maio

\section{Associação Catarinense de Medicina}

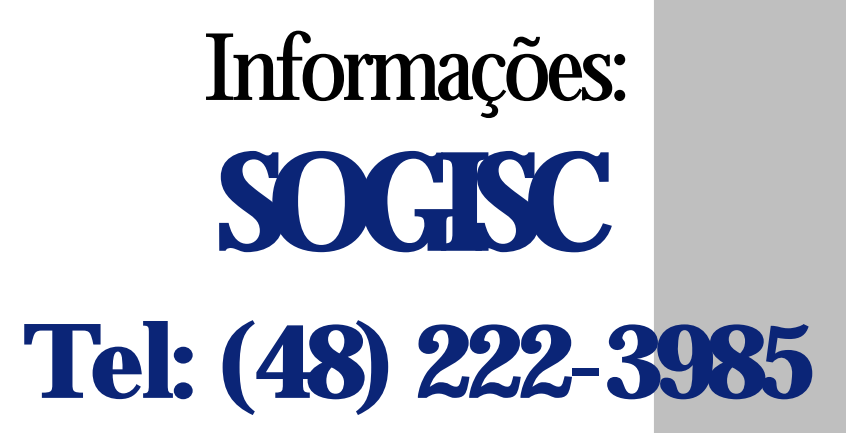

e-mail:oceanoeventos@ oceanoeventos.com.br 